Bodo v. PAPE, Oldenburg

\title{
Geometrisches Modellieren
}

Nach den Bildungsstandards der KMK ist das Modellieren eine der sechs Kernkompetenzen der Mathematik. Um der begrifflichen Klarheit willen beschränke ich mich auf das, was anderswo „Modellieren im engeren Sinn“ heißt, also das „Übersetzen“ einer Gegebenheit in Mathematik - hier Geometrie. Außen vor bleibt das - nicht selten stark übermächtige - „Arbeiten in dem jeweiligen mathematischen Modell“. Es geht um „Lösen anspruchsvoller mathematischer Problemaufgaben“. Aufgabenstellungen, bei denen die Herausforderung sich beschränkt auf den Einsatz eines einzigen Modells - etwa eines aktuellen Standardmodells - scheiden damit auch aus.

Zwei Thesen vorab:

- Der Computer ist ein Standardwerkzeug beim GM.

- Innerfachlich geht es zunächst um Figuren und Körper im Koordinatensystem, dann um Differentialgeometrie auf numerischer Basis.

Den Thesen kommt man näher, wenn man kurz einen Blick über die Fachdidaktik hinaus wagt: „Das geometrische Modellieren beschäftigt sich mit dem rechnergestützten Entwurf und der Manipulation geometrischer Formen.“ (Abramowski-Müller, 1991) Dabei gibt es zwei Ansätze: Das „Kombinatorische Modellieren“ mit Standardprimitiven und das „Differentialgeometrische Modellieren“. Hier dominieren approximierende Verfahren.

Wer inhaltlich ausgerichtet ist auf Lebensnähe, der wird sich auch im Bereich der mathematischen Werkzeuge entscheiden für ein Tool, das im Leben etabliert ist. MS-Excel („Office-Standard“) ist an den Schulen verfügbar, auch bei den Schülern sollte man es erwarten. Mit Excel kann man den Bereich der geometrischen Modellierungen in seinen Grundzügen in großer Breite und Vielfalt erschließen. Entscheidend ist dabei die Möglichkeit, das Angebot der Funktionen zu erweitern durch selbst erarbeitete Funktionsmakros (= Ketten von Wertzuweisungen) zu geometrischen Routinen. (Etwa: Schnittpunkte zweier Kreise)

\section{Modellierungsaufgaben}

Bei der Sichtung der Vorschläge zum Modellieren im MU im Hinblick auf ihre Eignung für den Unterrichtsalltag stößt man schnell auf ein Dilemma: Im Focus stehen Aufgabenstellungen, denen man gerecht werden kann eigentlich nur im Rahmen eines Projekts, möglichst fächerverbindend angelegt (,Authentischer Kontext, gesellschaftliches Interesse“): „Lohnt sich 
eine Fahrt zum Tanken nach Luxemburg?“ - „Versorgung beim Weltjugendtag in Köln“ - „Wie sollte man die Einkommensteuern festlegen?“ „Solarenergie in Deutschland - lohnt sich das?" Daneben stehen Aufgaben, in denen es um ein sehr kurzsinniges Abschätzen und Ankreuzen geht wie bei der Wassertank-Aufgabe aus den Bildungsstandards. Das Rechnen erweist sich nicht selten - angesichts der Unwägbarkeiten und Ungenauigkeiten der Ausgangsdaten - als aufgesetzt: „Lohnt sich die Abkürzung?“ aus den Bildungsstandards oder die „Zuckerhut"-Aufgabe

Im Rahmen der IQB-Aufgabe zu den Abraumhalden geht es um Höhenlinien einer Landschaft aus zwei Kegeln gleicher Höhe. Hier sollte man unter „Geometrischer Modellierung“ mehr erwarten als bloßes Ausscheiden abwegiger Vorgaben: Wie sieht es eigentlich aus bei Kegeln unterschiedlicher Höhe. Wie sieht es aus bei unterschiedlicher Neigung? Wie sehen insbesondere die „Tal“-Linien aus? Wie sieht das Geflecht der Tallinien aus, wenn man die Zahl der Kegel von zwei erhöht fünf?

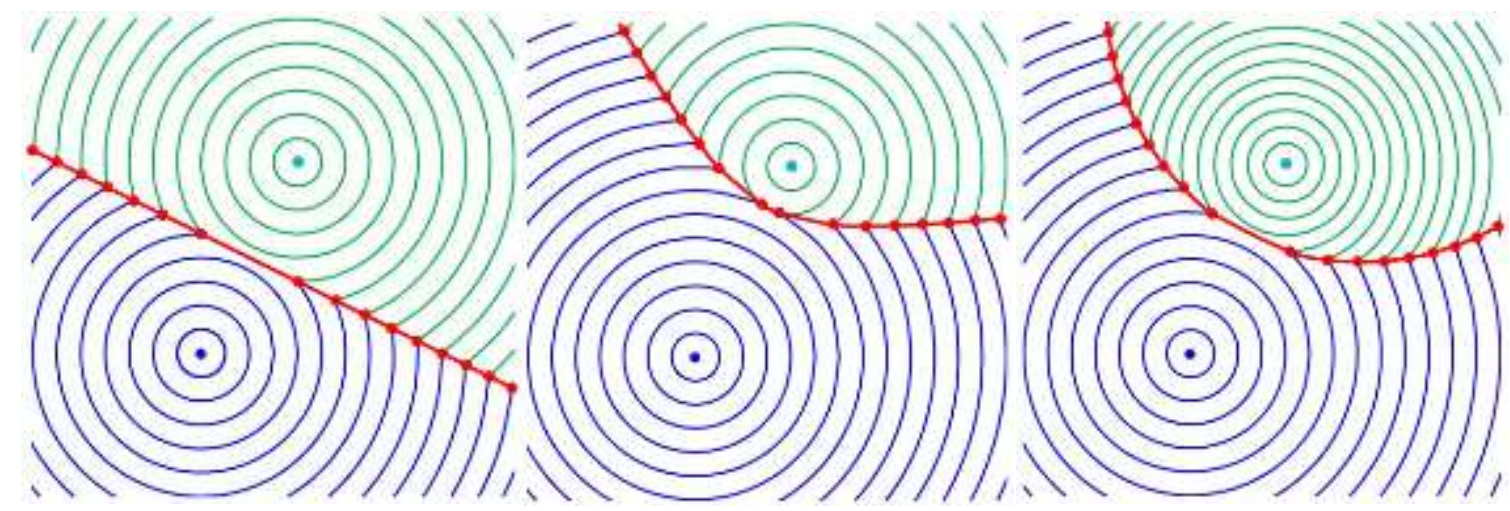

\section{Klassifikation der geometrischen Modellierungen}

Eine erste Einteilung nach der Vorgehensweise ist bereits ins Auge gefasst. Jeder der beiden Vorgehensweisen - „Elementargeometrisch“ und „Differentialgeometrisch“ - lässt sich eine Klasse typischer Objekte zuordnen. Weitergehend bietet sich hier eine Differenzierung nach der Zielsetzung an.

Zweck einer Modellierung kann auch die sehr direkte Nachbildung eines vorhandenen Objekts („Replikation“) sein - Warenzeichen, Parkettierung, Werk aus der Konkreten Kunst (Nees: „Schotter") - sowie das Design von etwas, das erst noch hergestellt werden soll. („Produktion“)

Neben die statischen Modellierungen tritt der Typ der kinematischen.

Dem geometrischen Modellieren zuzurechnen ist zweifelsfrei auch das Erstellen von Papier-, Drahtgitter-, Strohhalm- und Höhenschichtenmodellen. 


\section{Elementargeometrisches Modellieren}

Die elementargeometrische Modellierung erfolgt idealisierend aus ebenen Primitiven - Rechteck, Dreieck, Kreis - oder räumlichen: Quader, Würfel, Kugel, Kegel, Pyramide. Als Objekte drängen sich auf: Haus, Auto, Baum, Dach. Die Passung der Teile kann durchaus diffizil sein, so etwa beim Modellieren von Maßwerk. Approximieren durch Streckenzüge oder Polygone tritt als Standardverfahren hinzu.

Nicht nur im Primarschulbereich kommt diesem Verfahren Bedeutung zu. In der professionellen Anwendung deckt es ein breites Spektrum ab: Im Maschinenbau lassen sich 90\% der Bauteile auf diese Weise modellieren.

\section{Differentialgeometrisches Modellieren}

Zum einen geht es um Naturformen: Blüte, Ei, Phyloceras, Wasserstrahl, Kette, Muschel. Noch ergiebiger ist der Bereich „Artefakte“: Vasen, Gläser, Flaschen, Giebel, Brücken, Bögen bei Kirchen, Ziergitterelemente.

Im Wesentlichen geht es um eine Anpassung an eine Vorlage. Zum Einsatz kommen dabei höhere mathematische Objekte, in der Ebene zunächst Funktionsgraphen - Parabel, Polynomfunktion, Kettenlinie, Spline -, dann aber vor allem Kurven: Kegelschnitte, Spiralen, Zykloiden, Bezier-Kurven, Klotoiden. Im Raum greift man - etwa bei Modellierungen aus der Architektur - zurück auf Raumkurven und Funktionsflächen.

\section{Visualisierung}

Allgemein gesprochen zielt das geometrische Modellieren auf eine vereinfachte Wiedergabe einer augenfälligen Gegebenheit. Spricht man von „Visualisierung“, so hat man mehr im Auge. (Neubrand: „Zu den in der Geometrie authentisch und dennoch elementar darstellbaren Tätigkeiten gehören das Aufklären von Phänomenen, das Aufdecken verborgener Beziehungen und die Präzisierung qualitativer Beziehungen.") So kann es gehen um

- die Aufdeckung eines verborgenen Sachverhalts

- den Aufweis eines theoretischen Hintergrunds

- die Umsetzung eines abstrakten Sachverhalts

- eine Illustration eines imaginierten Sachverhalts

Die Darstellung von Spuren -beim Basketballwurf, beim Einparken oder bei Verfolgungsproblemen - wird man der ersten Kategorie zurechnen, die Hinterlegung der Katakaustik in einer Tasse mit Strahlenverläufen der zweiten. Beim Modellieren eines Stapels von überstehenden Bausteinen 
nimmt man das Unendliche in den Blick. Stilisierte Darstellungen - Herzen, Bäume - wird man der vierten Kategorie zurechnen.

\section{Kinematische Modelle}

Bei Modellierungsaufgaben sind in der Regel Daten vorgegeben, nicht sel-

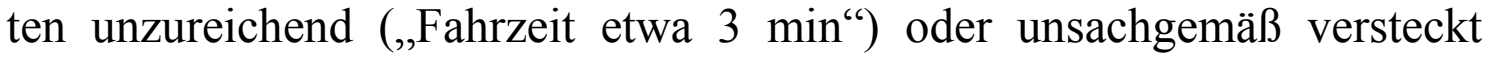
(,Geschwindigkeit $30 \mathrm{~km} / \mathrm{h}^{\text {“: }}$ Wie misst man das eigentlich?). In der Geometrie dagegen geht man aus von einer Abbildung. Der wichtigste Schritt der Modellierung besteht in der Wahl der Parameter. Der Übergang zu einem kinematischen Modell erfolgt problemlos über eine Variation der Werte einiger dieser Parameter über eine Zählschleife. In der Bewegung zeigt sich dann, ob die Wahl der Parameter günstig war. So geht die Validierung eines kinematischen Modells hinaus über den bloßen visuellen Vergleich mit der Vorlage. (Kriterium der „Zugfestigkeit“)

In der Literatur wird dieser Bereich abgedeckt mit Beispielen wie: Briefwaage, Kran, Bagger, Hubkolben, Garagentor, Einparken,

\section{Interdisziplinäres Modellieren, innerfachliches Modellieren}

$\mathrm{Zu}$ den klassischen geometrischen Modellen gehören die zum Planetensystem und zum Atom. Lichtstrahl und Elementarwelle sind weitere einschlägige geometrische Modelle aus der Physik. Bei der Modellierung einer Leiterschleife im Magnetfeld - „Elektromotor“ - wird man sogar ein aktuelles gesellschaftliches Interesse unterstellen dürfen.

Visualisierungen zur Erzeugung von Ortslinien sollten als Modellierungen einzustufen sein, und zwar ganz unabhängig davon, ob ein Sachverhalt aus der Realität widergespiegelt wird („Garagentor“: Zimmermannskonstruktion der Ellipse) oder nicht. Dasselbe gilt für Visualisierungen zur mechanischen Erzeugung von Kurven insgesamt („Cyklograph“: Rollkurven). Schließlich: Weiter tragende innermathematische Modellierungen - etwa mit Streckenzügen oder Treppen - können bei unserem Thema nicht ausgeschlossen bleiben.

\section{Bilanz}

Der Themenbereich ist umfassend, gehaltvoll und sehr attraktiv. Aber:

- GM und Unterrichtsalltag - passt das überhaupt zusammen?

- GM- und „PISA“-Aufgaben - passt das zusammen??

\section{Literatur}

Heinz Schumann: Rekonstruktives Modellieren in Dynamischen Geometriesystemen mathematica didactica 26 (2003), Bd. 2, 21-42 